\title{
WEAKLY NORMAL FILTERS AND THE CLOSED UNBOUNDED FILTER ON $P_{\kappa} \lambda$
}

\author{
YOSHIHIRO ABE
}

(Communicated by Thomas J. Jech)

\begin{abstract}
Assuming that $\kappa$ is supercompact and $\lambda$ is inaccessible, we present two isomorphic fine measures on $P_{\kappa} \lambda$ containing the closed unbounded filter. Some remarks on the (strongly) closed unbounded filter and weakly normal filters are added.
\end{abstract}

In the theory of $\kappa$-ultrafilters on a measurable cardinal $\kappa$, the closed unbounded filter (the club filter) plays an important role. For instance, Ketonen showed that any two distinct $\kappa$-ultrafilters containing the club filter are not isomorphic.

Weakly normal filters on a regular cardinal are also important. A filter is weakly normal iff it is a $p$-point containing the club filter. Every countably complete ultrafilter is minimal in the RK-ordering iff it is isomorphic to a weakly normal ultrafilter.

Jech is the first to introduce some combinatorial principles into $P_{\kappa} \lambda$ from the usual fields of $\kappa$. At first $P_{\kappa} \lambda$ seemed the same as $\kappa$. But it turned out to be more complicated. Menas proved that every normal measure on $P_{\kappa} \lambda$ where $\lambda$ is a strong limit with the cofinality less than $\kappa$ is isomorphic to a fine measure containing the club filter on $P_{\kappa} \lambda$. (See Proposition 12 in [9].) In [4], Gitik constructed a model in which there is a stationary subset of $P_{\kappa} \kappa^{+}$that cannot be split into $\kappa^{+}$disjointed stationary sets.

Applying Menas' result, we present two isomoprhic fine measures on $P_{\kappa} \lambda$ both of which contain the club filter under the hypothesis that $\kappa$ is supercompact and $\lambda$ is strongly inaccessible.

In [1] , a kind of fine measure on $P_{\kappa} \lambda$ investigated by Menas, was studied. By the embedding argument, it was pointed out that such a measure is not normal and can be weakly normal in suitable conditions. We take a combinatorial approach and show that filters of the same type do not contain a standard club set, indeed strongly closed unbounded. We extend the results in [1] on the weak normality of such a filter.

At last, some remarks on the relation between the RK-order and weakly normal fine measures, the strongly club filter and the partition property are added.

0. Definitions and notations. $\kappa$ is a regular uncountable cardinal and $\lambda$ is a cardinal $>\kappa$ throughout. $P_{\kappa} \lambda=\{x \subset \lambda:|x|<\kappa\}$. When we speak of a filter on $P_{\kappa} \lambda$ it is assumed to be $\kappa$-complete and fine, where $U$ is fine iff $\{x: \alpha \in x\} \in U$ for all $\alpha<\lambda$.

Received by the editors September 22, 1987, and in revised form, February 8, 1988.

1980 Mathematics Subject Classification (1985 Revision). Primary 04A20; Secondary 03E05. 
DEFINITION 0.1. $U$ is normal if every regressive function is constant on a set of positive measure. (We write $X \in U^{+}$if $X$ is positive measure.) $U$ is weakly normal if every regressive function is bounded by some $\gamma<\lambda$ on a set in $U$. We call $U$ a fine measure if it is an ultrafilter.

A subset $C$ of $P_{\kappa} \lambda$ is said to be unbounded if for each $a \in P_{\kappa} \lambda$ there is an $x \in C$ so that $a \subset x$. $\hat{a}$ denotes the set $\left\{x \in P_{\kappa} \lambda: a \subset x\right\}$. Thus $C$ is unbounded if $\hat{a} \cap C \neq 0$ for all $a \in P_{\kappa} \lambda$. $C$ is closed if $\bigcup A \in C$ whenever $A$ is a $\subset$-increasing chain of length $<\kappa$ in $C$. $C$ is strongly closed if $\bigcup A \in C$ for all $A \subset C$ with $|A|<\kappa$. The club filter $\mathrm{CF}_{\kappa \lambda}$ is the filter generated by the closed unbounded sets. The strongly club filter $\mathrm{SCF}_{\kappa \lambda}$ is the filter generated by the strongly closed unbounded sets.

Let $U$ be a fine measure on $P_{\kappa} \lambda$ and $f: P_{\kappa} \lambda \rightarrow P_{\kappa} \lambda$. The ultrafilter $f_{*}(U)$ defined by " $X \in f_{*}(U)$ if $f^{-1}(X) \in U$ " is a fine measure provided that $\{x: \alpha \in f(x)\} \in U$ for all $\alpha<\lambda$.

DEFINITION 0.2. Suppose that $U$ and $D$ are fine measures on $P_{\kappa} \lambda$. We write $U \leq D$ if $U=f_{*}(D)$ for some $f: P_{\kappa} \lambda \rightarrow P_{\kappa} \lambda$. $U$ and $D$ are isomorphic $(U \cong D)$ if $U=f_{*}(D)$ and $f$ is one-to-one on a set $X \in D$. D is minimal in the RK-order if $D$ is isomorphic to all $U \leq D$.

DEFINTION 0.3. Suppose that $f$ is an ordinal valued function with domain $P_{\kappa} \lambda$. $f$ is the first function of $U$ if $\{x: f(x)>\gamma\} \in U$ for any $\gamma<\lambda$, and $\{x: g(x)<\gamma\} \in U$ for some $\gamma<\lambda$ whenever $\{x: g(x)<f(x)\} \in U$.

The first function tells us whether a fine measure is minimal or not under the certain assumption on $\lambda$.

DEFINITION 0.4. A fine measure $U$ has the partition property if every $F:\left[P_{\kappa} \lambda\right]^{2}$ $=\left\{\{x, y\}: x, y \in P_{\kappa} \lambda\right.$ and $\left.x \subsetneq y\right\} \rightarrow 2$ has a homogeneous set in $U$. ( $A$ is homogeneous for $F$ if there is a $k<2$ so that for all $x, y \in A$ with $x / \subsetneq y, F(\{x, y\})=k$.)

1. Isomorphic fine measures. In this section, $\lambda$ is a fixed inaccessible cardinal greater than $\kappa$, a supercompact. We shall present two isomorphic fine measures including $\mathrm{CF}_{\kappa \lambda}$. Though we extend the result of Menas, we have to start from it.

LEMMA 1.1 (MENAS [9]). Let $\delta$ be a strong limit cardinal with the cofinality less than $\kappa$. Then every normal measure on $P_{\kappa} \delta$ is isomorphic to a nonnormal fine measure containing $\mathrm{CF}_{\kappa \delta}$.

Let $A=\{\delta: \kappa<\delta<\lambda, \delta$ is strong limit, $\operatorname{cf}(\delta)<\kappa\}$. For each $\delta \in A$, there is a function $q^{\delta}: P_{\kappa} \delta \rightarrow P_{\kappa} \delta$ so that $\mathrm{CF}_{\kappa \delta} \subset q_{*}^{\delta}\left(U_{\delta}\right) \cong U_{\delta}$ where $U_{\delta}$ is a normal measure on $P_{\kappa} \delta$. We shall sum up these $U_{\delta}$ 's and $q_{*}^{\delta}\left(U_{\delta}\right)$ 's with a suitable ultrafilter on $\lambda$.

LEMMA 1.2. There exists a $\kappa$-complete ultrafilter on $\lambda$ including $\{A\} \cup \mathrm{CF}_{\lambda}$. $\left(\mathrm{CF}_{\lambda}\right.$ is the club filter on $\lambda$.)

Proof. Since $\lambda$ is inaccessible, $A$ is stationary. Hence we have a $\lambda$-complete filter $E=\{X \subset \lambda: A-X$ is not stationary $\}$. It is easily seen that $\{A\} \cup \mathrm{CF}_{\lambda} \subset E$. Then the strong compactness of $\kappa$ gives us a $\kappa$-complete ultrafilter $D$ extending $E$.

We use the above $D$. Define $F_{1}$ and $F_{2}$ by

$$
\begin{array}{ll}
X \in F_{1} & \text { if } X \subset P_{\kappa} \lambda \text { and }\left\{\delta \in A: X \cap P_{\kappa} \delta \in U_{\delta}\right\} \in D, \\
X \in F_{2} & \text { if } X \subset P_{\kappa} \lambda \text { and }\left\{\delta \in A: X \cap P_{\kappa} \delta \in q_{*}^{\delta}\left(U_{\delta}\right)\right\} \in D .
\end{array}
$$

$F_{1}$ and $F_{2}$ are fine measures on $P_{\kappa} \lambda$. We want to show that they are isomorphic and contain $\mathrm{CF}_{\kappa \lambda}$. The next is an easy but key lemma. 
LEMMA 1.3. Assume that $\operatorname{cf}(\eta)<\kappa$ and $U$ is a fine measure on $P_{\kappa} \eta$. Then $\left\{x \in P_{\kappa} \eta: \sup (x)=\eta\right\} \in U$.

ProOF. Let $\left\{\eta_{\alpha}: \alpha<\operatorname{cf}(\eta)\right\}$ be a cofinal subset of $\eta$. Since $U$ is fine, $\left\{x: \eta_{\alpha} \in\right.$ $x\} \in U$ for each $\alpha<\operatorname{cf}(\eta)$. Using the $\kappa$-completeness of $U$ and the fact that $\operatorname{cf}(\eta)<\kappa$, we get $\left\{x: \eta_{\alpha} \in x\right.$ for every $\left.\alpha<\operatorname{cf}(\eta)\right\} \in U$.

Corollary 1.4. For every $\delta \in A,\left\{x \in P_{\kappa} \delta: \sup (x)=\delta\right\} \in U_{\delta}$ and $\{x \in$ $\left.P_{\kappa} \delta: \sup \left(q^{\delta}(x)\right)=\delta\right\} \in U_{\delta}$.

PROOF. Since $q_{*}^{\delta}\left(U_{\delta}\right)$ is also a fine measure on $P_{\kappa} \delta$ and $\operatorname{cf}(\delta)<\kappa,\{x: \sup (x)=$ $\delta\} \in q_{*}^{\delta}\left(U_{\delta}\right)$. This is equivalent to $\left\{x: \sup \left(q^{\delta}(x)\right)=\delta\right\} \in U_{\delta}$.

For $x \in P_{\kappa} \lambda$, let $\delta_{x}=$ the least member of $A$ such that $x \in P_{\kappa} \delta$. And $q: P_{\kappa} \lambda \rightarrow$ $P_{\kappa} \lambda$ is defined by

By our construction,

$$
q(x)=q^{\delta_{x}}(x)
$$

LEMMA 1.5. For every $\delta \in A,\left\{x \in P_{\kappa} \delta: \delta_{x}=\delta\right\} \in U_{\delta}$; hence $\{x: q(x)=$ $\left.q^{\delta}(x)\right\} \in U_{\delta}$.

We can see that $F_{1}$ and $F_{2}$ are isomorphic.

LEMMA 1.6. $q$ is one-to-one on a set in $F_{1}$.

PROOF. Let $B_{\delta} \in U_{\delta}$ be such that $q^{\delta}$ is one-to-one on $B_{\delta}$. We have already known that $C_{\delta}=\left\{x \in B_{\delta}: q(x)=q^{\delta}(x), \sup \left(q^{\delta}(x)\right)=\sup (x)=\delta\right\} \in U_{\delta}$. Hence $C=\bigcup\left\{C_{\delta}: \delta \in A\right\}$ is a member of $F_{1}$.

Suppose that $x, y \in C$ and $q(x)=q(y)$. There is a $\delta \in A$ such that $\delta=$ $\sup (x)=\sup (q(x))=\sup (q(y))=\sup (y)$. Since $x$ and $y$ are in the same $C_{\delta}$ and $q \uparrow C_{\delta}=q^{\delta} \uparrow C_{\delta}$ is one-to-one, we have $x=y$. Thus $q$ is one-to-one on $C \in F_{1}$.

LEMMA 1.7. $F_{2}=q_{*}\left(F_{1}\right)$.

ProOF. Recall that $X \in F_{2}$ iff $\left\{\delta \in A: X \cap P_{\kappa} \delta \in q_{*}^{\delta}\left(U_{\delta}\right)\right\} \in D$, and that $X \cap P_{\kappa} \delta \in q_{*}^{\delta}\left(U_{\delta}\right)$ is equivalent to $\left\{x \in P_{\kappa} \delta: q^{\delta}(x) \in X \cap P_{\kappa} \delta\right\} \in U_{\delta}$. By 1.5, the last paraphrase is the same as $\left\{x \in P_{\kappa} \delta: q(x) \in X\right\} \in U_{\delta}$.

Let $Y=\left\{x \in P_{\kappa} \lambda: q(x) \in X\right\}$. We have shown that $X \in F_{2}$ is equivalent to $\left\{\delta \in A: Y \cap P_{\kappa} \delta \in U_{\delta}\right\} \in D$. The latter says that $Y \in F_{1}$ and $X \in q_{*}\left(F_{1}\right)$. Hence $X \in F_{2}$ iff $X \in q_{*}\left(F_{1}\right)$.

What is left to show is that both $F_{1}$ and $F_{2}$ contain $\mathrm{CF}_{\kappa \lambda}$. Note that $\{\delta<$ $\left.\lambda: X \cap P_{\kappa} \delta \in \mathrm{CF}_{\kappa \delta}\right\} \in \mathrm{CF}_{\lambda}$ for every $X \in \mathrm{CF}_{\kappa \lambda}$.

LEMMA 1.8. $\mathrm{CF}_{\kappa \lambda} \subset F_{1} \cap F_{2}$.

Proof. Suppose that $X \in \mathrm{CF}_{\kappa \lambda}$. Then $X^{\prime}=\left\{\delta<\lambda: X \cap P_{\kappa} \delta \in \mathrm{CF}_{\kappa \delta}\right\} \in$ $\mathrm{CF}_{\lambda} \subset D$. Since $U_{\delta}$ and $q_{*}^{\delta}\left(U_{\delta}\right)$ contain $\mathrm{CF}_{\kappa \delta}, X \cap P_{\kappa} \delta$ belongs to both $U_{\delta}$ and $q_{*}^{\delta}\left(U_{\delta}\right)$ for all $\delta \in X^{\prime}$. Hence $X \in F_{1} \cap F_{2}$.

Now we are done.

THEOREM 1.9. If $\lambda$ is a strongly inaccessible cardinal greater than $\kappa$ a supercompact, there are two distinct isomorphic fine measures on $P_{\kappa} \lambda$ containing the club filter.

The author does not know whether a normal measure on $P_{\kappa} \lambda$ is isomorphic to a fine measure containing $\mathrm{CF}_{\kappa \lambda}$ under the same assumption. It is also still open 
whether two fine measures can be isomorphic for a successor cardinal $\lambda$. The case that $\lambda$ is not strong limit is also open.

2. $\mathrm{SCF}_{\kappa \lambda}$, prestationary sets and the partition property. For the subsets of regular uncountable cardinals, the situation is simple. That is, $S \subset \kappa$ is stationary iff for any regressive function $f$ on $S$, there is an unbounded set $T \subset S$ on which $f$ is constant. But this does not hold for the subsets of $P_{\kappa} \lambda$.

In this section, $\kappa$ is a regular uncountable cardinal and $\lambda>\kappa$. We begin by Menas' invention again.

PROPOSITION 2.1 (MENAS [8]). There is a nonstationary subset $S$ of $P_{\kappa} \lambda$ such that every regressive function is constant on an unbounded subset of $S$.

DEFINITION 2.2. We call such a set $S$ "prestationary".

Menas characterized $S$ 'stationary" as follows:

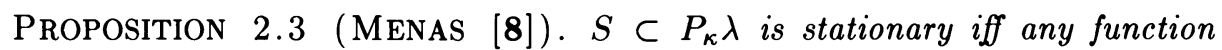
$f: S \rightarrow \lambda \times \lambda$ so that $f(y) \in y \times y$ for all $y$ in $S$, is constant on some unbounded $T \subset S$.

In the spirit of Proposition 2.3, we can express stationarity using prestationarity.

PROPOSITION 2.4. If $S \subset P_{\kappa} \lambda$ is prestationary and every regressive function is constant on a prestationary $T \subset S$, then $S$ is stationary.

ProOF. Let $f: S \rightarrow \lambda \times \lambda, f_{1}, f_{2}: S \rightarrow \lambda$ so that $f(y) \in y \times y$ for all $y \in S$ and $f(y)=\left(f_{1}(y), f_{2}(y)\right)$. Since $f_{1}(y) \in y$ for all $y \in S$, there is a prestationary $T_{1} \subset S$ on which $f_{1}$ is constant. Again by the fact that $f_{2}(y) \in y$ for every $y \in T_{1}$ that is prestationary, there is an unbounded $T_{2} \subset T_{1}$ so that $f_{2}\left\lceil T_{2}\right.$ is constant. Then $f \uparrow T_{2}$ is constant.

The stationary subsets are the sets which have nonempty intersection with every closed unbounded set. Now we characterize the prestationary sets with $\mathrm{SCF}_{\kappa \lambda}$. First recall the theorem for $\mathrm{SCF}_{\kappa \lambda}$ in Carr [3].

LEMMA 2.5 (CARR). $C \in \mathrm{SCF}_{\kappa \lambda}$ iff there is a sequence of sets in $P_{\kappa} \lambda$, $\left\langle x_{\alpha} \mid \alpha<\lambda\right\rangle$ so that $\Delta\left\langle\hat{x}_{\alpha} \mid \alpha<\lambda\right\rangle=\left\{y: x_{\alpha} \subset y\right.$ for all $\left.\alpha \in y\right\} \subset C$.

Proposition 2.6. $S \subset P_{\kappa} \lambda$ is prestationary iff $S \cap C \neq 0$ for all $C \in \mathrm{SCF}_{\kappa \lambda}$.

Proof. Suppose that $S$ is prestationary and $S \cap C=0$ for some $C \in \mathrm{SCF}_{\kappa \lambda}$. By 2.5, there is a sequence $\left\langle x_{\alpha} \mid \alpha<\lambda\right\rangle$ so that $\Delta\left\langle\hat{x}_{\alpha} \mid \alpha<\lambda\right\rangle \subset C$. For every $x \in S$, there exists an $\alpha \in x$ such that $x_{\alpha} \not \subset x$. Since $S$ is prestationary, there is an ordinal $\gamma$ so that $\left\{x \in S: x_{\gamma} \not \subset x\right\}$ is unbounded. Contradiction.

For the converse, assume that $S \cap C \neq 0$ for all $C \in \mathrm{SCF}_{\kappa \lambda}$ and $S$ is not prestationary. There is a regressive function $f$ such that for every $\alpha<\lambda$ there is an $a_{\alpha} \in P_{\kappa} \lambda$ so that $\{x \in S: f(x)=\alpha\} \cap \hat{a}_{\alpha}=0$. Let $C=\Delta\left\{\hat{a}_{\alpha} \mid \alpha<\lambda\right\}$; then $C \in \mathrm{SCF}_{\kappa \lambda}$. Pick an $x \in C \cap S$ and suppose that $f(x)=\alpha$. Since $\alpha \in x$ and $x \in C$, $a_{\alpha} \subset x$. Then $f(x) \neq \alpha$ by the definition of $a_{\alpha}$. This is absurd.

We connect the above fact to the partition property of fine measures.

COROLLARY 2.7. If $U$ is a fine measure with the partition property assigning measure one to the strongly club sets, then $U$ is normal.

This is really Proposition 11 in Menas [9], where he proved it for the club sets version. Menas' proof is applicable in our case as well. 
3. Weakly normal filters on $P_{\kappa} \lambda$. For weakly normal filters on $\kappa$ regular, see Kanamori [7]. We briefly review the basic facts.

PROPOSITION 3.1. For any filter on $\kappa$, the following are equivalent.

(i) $U$ is weakly normal.

(ii) Every filter extension of $U$ is weakly normal.

(iii) If $\left\{X_{\alpha}: \alpha<\kappa\right\}$ are sets of positive measure such that $X_{\beta} \subset X_{\alpha}$ whenever $\alpha<\beta$, then $\Delta\left\{X_{\alpha}: \alpha<\kappa\right\}=\left\{\alpha<\kappa: \alpha \in X_{\beta}\right.$ for all $\left.\beta<\alpha\right\}$ has a positive measure.

(iv) $U$ is a p-point filter extending $\mathrm{CF}_{\kappa}$. ( $U$ is a p-point if every function $f: \kappa \rightarrow \kappa$ such that $\kappa-f^{-1}(\{\alpha\}) \in U$ for all $\alpha<\kappa$ is $<\kappa$ to one on some $X \in U$.)

It is natural to ask whether the same thing happens to filters on $P_{\kappa} \lambda$. We easily get that (i) (iii) are also equivalent for any filter on $P_{\kappa} \lambda$. (Note that $\Delta\left\{X_{\alpha}: \alpha<\lambda\right\}=\left\{x \in P_{\kappa} \lambda: x \in X_{\alpha}\right.$ for all $\left.\alpha \in X\right\}$.)

But for (iv), the author only knows the following.

PROPOSITION 3.2. (i) Suppose that $U$ is weakly normal. If $f$ is a function with the domain $P_{\kappa} \lambda$ and $\{x: f(x)>\alpha\} \in U^{+}$for all $\alpha<\lambda$, then there is a set $X$ of positive measure so that $X \cap f^{-1}(\{\alpha\}) \subset P_{\kappa} \alpha$ for all $\alpha<\lambda$.

(ii) Suppose that $U$ extends $\mathrm{SCF}_{\kappa \lambda}$ and for any $\alpha<\lambda$ there is an $X \in U^{+}$such that $X \cap f^{-1}(\{\alpha\}) \subset P_{\kappa} \beta$ for some $\beta<\lambda$ whenever $f$ satisfies $\{x: f(x)>\gamma\} \in U^{+}$ for all $\gamma<\lambda$. Then $U$ is weakly normal.

ProOF. (i) Let $X_{\xi}=\{x: f(x)>\xi\}$ for each $\xi<\lambda$. Then $X_{\xi} \in U^{+}$and $X_{\eta} \subset X_{\xi}$ if $\xi<\eta$. Now $\Delta\left\{X_{\xi}: \xi<\lambda\right\} \in U^{+}$by (iii). If $x \in \Delta\left\{X_{\xi}: \xi<\lambda\right\}$ and $f(x)=\alpha$, then $\xi<\alpha$ for all $\xi \in x$. Hence $x \subset \alpha$.

(ii) Suppose that $f$ is a regressive function on $P_{\kappa} \lambda$. Since $U$ extends $\mathrm{SCF}_{\kappa \lambda}$, every $X$ of positive measure is prestationary. Hence there is an $\alpha<\lambda$ so that $X \cap f^{-1}(\{\alpha\})$ is unbounded. By our hypothesis, $\{x: f(x)<\gamma\} \in U$ for some $\gamma<\lambda$.

The question left is whether every weakly normal filter extends $\mathrm{CF}_{\kappa \lambda}$ or $\mathrm{SCF}_{\kappa \lambda}$. In [1], the fine measure investigated by Menas was revisited and shown to be nonnormal. We again observe it and get more information, which gives a negative answer to the question. The author wishes to express his gratitude to A. Blass whose advice led to a simplified proof. We concentrate on a filter defined below. We assume that $\kappa$ is a regular limit cardinal.

Let $\left\langle U_{\alpha} \mid \alpha<\kappa\right\rangle$ be a sequence of fine filters on $P_{\alpha} \lambda$ and $D$ be a $\kappa$-complete uniform filter on $\kappa$. Then a fine filter $U$ is defined by $X \in U$ if $X \subset P_{\kappa} \lambda$ and $\left\{\alpha<\kappa: X \cap P_{\alpha} \lambda \in U_{\alpha}\right\} \in D$.

THEOREM 3.3 (INSPIRED BY BLASS). U does not extend $\mathrm{SCF}_{\kappa \lambda}$ hence is nonnormal.

Proof. Let $C=\left\{x \in P_{\kappa} \lambda: x \cap \kappa\right.$ is an ordinal $\}$. Then $C$ is strongly closed unbounded. We shall show that $C \cap P_{\alpha} \lambda$ is not unbounded for all $\alpha<\kappa$. If $x \in C \cap P_{\alpha} \lambda$ and $\alpha^{+} \in x$, then $\alpha^{+} \subset x$. But this contradicts $|x|<\alpha$. Hence $\alpha^{+} \notin x$ for all $x \in C \cap P_{\alpha} \lambda$ and $C \cap P_{\alpha} \lambda \notin U_{\alpha}$. Thus $C \notin U$. Note that $\alpha^{+}<\kappa<\lambda$ since $\kappa$ is a limit cardinal.

For certain $A \subset \kappa$ we have a strongly club set which is not unbounded for any $\alpha \in A$. More precisely; 
Proposition 3.4. Suppose that $\lambda^{<\kappa}=\lambda$ and $A \subset \kappa$. There is a $C \in \mathrm{SCF}_{\kappa \lambda}$ so that if $\alpha \in A$ and $\sup (A \cap \alpha) \neq \alpha$, then $C \cap P_{\alpha} \lambda$ is not unbounded.

PROOF. Let $\left\{x_{z}: \xi<\lambda\right\}$ be an enumeration of $P_{\kappa} \lambda$ and $\alpha_{z}=$ the least member of $A>|x|$. Then, we pick a $y_{z} \supset x$ with $\left|y_{z}\right| \geq \alpha_{\xi}^{+}$. Finally, $C=\Delta\left\langle\hat{y}_{z} \mid \xi<\lambda\right\rangle$.

Suppose that $\alpha \in A$ and $\sup (A \cap \alpha) \neq \alpha$. Then $\alpha=\alpha_{z}$ for some $x_{z}$. Assume that there exists an $x \in C \cap P_{\alpha} \lambda$ with $\xi \in x$. By our definition of $C, x \supset y_{z}$. This implies $|x| \geq\left|y_{z}\right| \geq \alpha_{\xi}^{+}>\alpha$ contradicting $x \in P_{\alpha} \lambda$. Hence $\left(C \cap P_{\alpha} \lambda\right) \cap\{\hat{\xi}\}=0$.

Now we turn to the weak normality of $U$ under the assumption that $U_{\alpha}$ is weakly normal for all $\alpha<\kappa$, and improve Proposition 2.4 in [1] by a simple argument. In the next theorem, $\kappa$ is not necessarily a limit cardinal in (i) and (iii).

THEOREM 3.5. (i) If $\operatorname{cf}(\lambda)>\kappa$, then $U$ is weakly normal.

(ii) If $\operatorname{cf}(\lambda)=\kappa$, then $U$ is not weakly normal.

(iii) If $\operatorname{cf}(\lambda)<\kappa$ and (a) or (b) is satisfied, then $U$ is weakly normal.

(a) $U$ is an ultrafilter.

(b) $D$ is $\operatorname{cf}(\lambda)$-descendingly complete. That is; if $\left\langle X_{\xi} \mid \xi<\operatorname{cf}(\lambda)\right\rangle$ is a sequence of positive measure such that $X_{\eta} \subset X_{\xi}$ whenever $\xi<\eta$, then $\bigcap\left\{X_{\xi}: \xi \operatorname{cf}(\lambda)\right\} \neq 0$. (Note that $D$ is not required to be an ultrafilter.)

Proof. Suppose that $f(x) \in x$ for every $x \in P_{\kappa} \lambda$.

(i) For $\alpha<\kappa, \delta_{\alpha}$ is an ordinal $<\lambda$ such that $\left\{x \in P_{\alpha} \lambda\right.$ : $\left.f(x)<\delta_{\alpha}\right\} \in U_{\alpha}$. Since $\operatorname{cf}(\lambda)>\kappa, \delta=\sup \left(\left\{\delta_{\alpha}: \alpha<\kappa\right\}\right)<\lambda$. Obviously $\left\{x \in P_{\kappa} \lambda: f(x)<\delta\right\} \in U$.

(ii) Let $\left\{\lambda_{\alpha}: \alpha<\kappa\right\}$ be a cofinal subset of $\lambda$ and $\lambda_{\alpha}<\lambda_{\beta}$ if $\alpha<\beta$. For each $\alpha<\kappa,\left\{x \in P_{\alpha} \lambda: \lambda_{\alpha} \in x\right.$ and $\left.\lambda_{|x|}<\lambda_{\alpha}\right\} \in U_{\alpha}$. Hence we have $\left\{x \in P_{\kappa} \lambda: x-\lambda_{|x|} \neq\right.$ $0\} \in U$.

So, there is a function $g: P_{\kappa} \lambda \rightarrow \lambda$ such that $g(x) \in x$ and $g(x)>\lambda_{|x|}$ for almost all $x(\bmod U)$. For any $\alpha<\kappa$, we know that $\left\{x \in P_{\alpha} \lambda: x \supset \alpha^{+}\right\} \in U$ and then $\left\{x: \lambda_{|x|}>\lambda_{\alpha}\right\} \in U$. Hence $\left\{x \in P_{\kappa} \lambda: g(x)>\lambda_{\alpha}\right\} \in U$ for every $\alpha<\kappa$. We are done because $g$ is an unbounded regressive function.

(iii) Suppose that (a) holds. We already showed in Lemma 1.3 that every fine measure on $P_{\kappa} \lambda$ is weakly normal if $\operatorname{cf}(\lambda)<\kappa$. In fact,

Fact 3.6. A fine measure is weakly normal iff its first function maps $x$ to $\sup (x)$. (We denote such a function by Sup.)

When (b) holds, let $\left\{\lambda_{\alpha}: \alpha<\delta\right\}$ be a cofinal subset of $\lambda$ with $\delta=\operatorname{cf}(\lambda)$ so that $\lambda_{\alpha}<\lambda_{\beta}$ if $\alpha<\beta$. Suppose that $\left\{x \in P_{\kappa} \lambda: f(x)<\lambda_{\alpha}\right\} \notin U$ for all $\alpha<\delta$. Then $\left\{\xi<\kappa:\left\{x \in P_{\xi} \lambda: f(x)<\lambda_{\alpha}\right\} \in U_{\xi}\right\} \notin D$ for any $\alpha<\delta$. Hence

$$
C_{\alpha}=\left\{\xi<\kappa:\left\{x \in P_{\xi} \lambda: f(x)<\lambda_{\alpha}\right\} \notin U_{\xi}\right\} \in D^{+} .
$$

If $\alpha<\beta$, then $\left\{x \in P_{\xi} \lambda: f(x)<\lambda_{\beta}\right\} \notin U_{\xi}$ implies $\left\{x \in P_{\xi} \lambda: f(x)<\lambda_{\alpha}\right\} \notin U_{\xi}$ since $\lambda_{\alpha}<\lambda_{\beta}$. So, $C_{\beta} \subset C_{\alpha}$. Then $C=\bigcap\left\{C_{\alpha}: \alpha<\delta\right\} \neq 0$.

Pick a $\xi \in C$. $\left\{x \in P_{\xi} \lambda: f(x)<\lambda_{\alpha}\right\} \notin U_{\xi}$ for any $\alpha<\delta$. This contradicts the hypothesis that $U_{\xi}$ is weakly normal.

Note that a filter $F$ on $P_{\kappa} \lambda$ is weakly normal if it is $\operatorname{cf}(\lambda)$-descendingly complete.

Combining Theorems 3.3 and 3.5, we have;

COROLLARY 3.7. There is a weakly normal filter which does not extend $\mathrm{SCF}_{\kappa \lambda}$.

Jech [5] and Carr [3] showed that $\mathrm{CF}_{\kappa \lambda}$ is the minimal normal filter. Is there a nice analogue for weakly normal filter? Or, what is the consistency of weakly normal filters? (Note here we assume that any filter is fine and $\kappa$-complete.) 
4. Weakly normal fine measures and the RK-ordering. In this section, $\kappa$ is a fixed strongly compact cardinal. We observe the weak normality in view of the RK-ordering. First we review the fact established by Menas in [8].

THEOREM 4.1 (MENAS). (i) If $\operatorname{cf}(\lambda)<\kappa$ or $\lambda$ is regular, then every normal measure on $P_{\kappa} \lambda$ is minimal.

(ii) If $\lambda$ is regular and the first function of $U$ is one-to-one on a set of measure one, then $U$ is minimal.

We hope that every weakly normal measure is minimal as in the theory of uniform ultrafilters on a regular cardinal. In fact any minimal fine measure is isomorphic to a weakly normal measure.

PROPOSITION 4.2. Every fine measure has a weakly normal measure below it.

ProOF. Let $U$ be a fine measure and $g$ its first function. Define $f: P_{\kappa} \lambda \rightarrow P_{\kappa} \lambda$ by $f(x)=x \cap g(x)$.

By an easy observation, $\{x: \alpha \in f(x)\} \in U$ for all $\alpha<\lambda$ and $f_{*}(U)$ is a fine measure.

Suppose that $\{x: f(x) \in x\} \in f_{*}(U)$. It means that $\{x: h \circ f(x) \in x \cap g(x)\} \in U$. Since $g$ is the first function of $U$, we have $\{x: h \circ f(x)<\gamma\} \in U$ for some $\gamma<\lambda$. Hence $\{x: h(x)<\gamma\} \in f_{*}(U)$.

The next fact appeared already in [8] implicitly.

PROPOSITION 4.3. Let $\lambda$ be regular and $U$ a fine measure on $P_{\kappa} \lambda . U$ is minimal iff its first function is one-to-one on a set $X \in U$.

ProOF. Let $\left\{A_{\lambda}(\alpha): \alpha<\lambda\right\}$ be a partition of $\{\alpha<\lambda: \operatorname{cf}(\alpha)=\omega\}$ into disjointed stationary subsets. Let $f$ be the first function and define $q$ by $q(x)=\{\alpha<$ $f(x): A_{\lambda}(\alpha) \cap f(x)$ is stationary in $\left.f(x)\right\}$. Then $q_{*}(U)$ is a minimal fine measure (Theorem 2.14 in [8]).

Suppose that $U$ is minimal. $q \backslash X$ is one-to-one for some $X \in U$. But $q(x)=q(y)$ if $f(x)=f(y)$. Hence $f \uparrow X$ is one-to-one.

COROLLARY 4.4. A weakly normal measure on $P_{\kappa} \lambda$ with $\lambda$ regular is minimal iff Sup is one-to-one on a set of measure one.

A filter $F$ on a regular cardinal $\rho$ is called a $q$-point if every $<\rho$ to one function from $\rho$ to $\rho$ is one-to-one on a set $X \in F$. It is known that any filter extending $\mathrm{CF}_{\rho}$ is a $q$-point. $\mathrm{SCF}_{\kappa \lambda}$ also plays a role on the minimality of weakly normal measures.

PROPOSITION 4.5. Let $\lambda$ be regular. If $U$ is a minimal fine measure on $P_{\kappa} \lambda$ that is not weakly normal, then $\mathrm{SCF}_{\kappa \lambda} \not \subset U$.

PrOOF. Let $f$ be the first function. By our assumption, there is a set $X \in U$ so that $f \uparrow X$ is one-to-one and $f(x)<\sup (x)$ for all $x \in X$.

Suppose that $\mathrm{SCF}_{\kappa \lambda} \subset U$. Then $X$ is prestationary. For $x \in X$, set $g(x)=$ the least member of $x$ greater than $f(x)$. There is an unbounded set $Y \subset X$ such that $g^{\prime \prime} Y=\{\gamma\}$ for some $\gamma<\lambda$. Thus, $f^{\prime \prime} Y \subset \gamma$ and $|Y|=\lambda^{<\kappa}>\gamma$, which contradicts the fact that $f \uparrow Y$ is one-to-one. 
COROLLARY 4.6. Let $\lambda$ be regular. If $U$ is normal and $f_{*}(U) \supset \mathrm{SCF}_{\kappa \lambda}$, then $f_{*}(U)$ is weakly normal and $\{x: \sup (f(x))=\sup (x)\} \in U$.

COROLLARY 4.7. For any regular $\lambda>\kappa$, there is a nonminimal fine measure extending $\mathrm{CF}_{\kappa \lambda}$.

ProOF. Let $A=\{\alpha<\lambda: \operatorname{cf}(\alpha)<\kappa\}$ which is stationary in $\lambda$. We repeat the construction in $\S 1$.

There is a $\kappa$-complete ultrafilter on $\lambda, D \supset \mathrm{CF}_{\lambda} \bigcup\{A\}$. For each $\alpha \in A$, fix a fine filter $U_{\alpha}$ on $P_{\kappa} \alpha$ extending $\mathrm{CF}_{\kappa \alpha}$, and define $U$ by

$$
X \in U \quad \text { iff }\left\{\alpha<\lambda: X \cap P_{\kappa} \alpha \in U_{\alpha}\right\} \in D .
$$

Then $U$ is a fine measure extending $\mathrm{CF}_{\kappa \lambda}$.

We shall see that $U$ is not weakly normal, hence nonminimal by Proposition 4.5.

Since $A \in D, D$ is not normal. Thus there is a function $g$ so that $[g]_{D}=\lambda$ and $\{\alpha<\lambda: g(\alpha)<\alpha\} \in D$.

For $x \in P_{\kappa} \lambda$, let $\alpha_{x}=$ the least $\alpha$ such that $x \in P_{\kappa} \alpha$ and $f(x)=g\left(\alpha_{x}\right)$. For every $\alpha \in A,\left\{x \in P_{\kappa} \alpha: f(x)<\sup (x)\right\} \in U_{\alpha}$ since $\left\{x: \alpha_{x}=\alpha=\sup (x)\right\} \in U_{\alpha}$. Let $h(x)=$ the least member of $x$ greater than $f(x)$. $h$ is a regressive function on a set in $U$.

Pick a $\gamma<\lambda$. Then $B=\{\alpha \in A: \gamma<g(\alpha)\} \in D$. For all $\alpha \in B,\{x \in$ $\left.P_{\kappa} \alpha: f(x)=g\left(\alpha_{x}\right)=g(\alpha)\right\} \in U_{\alpha}$ and $\left\{x \in P_{\kappa} \alpha: f(x)>\gamma\right\} \in U_{\alpha}$. Hence $\{x \in$ $\left.P_{\kappa} \lambda: \gamma<f(x)\right\} \in U$. It shows that Sup is not the least function.

On the other hand, we have a minimal fine measure which is weakly normal and does not extend $\mathrm{SCF}_{\kappa \lambda}$. We recall the fine measure in $\S 3$. Suppose that $\lambda$ is regular and $\left\langle U_{\alpha} \mid \alpha<\kappa\right\rangle$ is a sequence of normal measures on $P_{\alpha} \lambda$ and $D$ is a normal measure on $\kappa$. Define $U$ by

$$
X \in U \quad \text { iff }\left\{\alpha<\kappa: X \cap P_{\alpha} \lambda \in U_{\alpha}\right\} \in D .
$$

Following the argument of $3.1,3,4$ in [10], we get

LEMMA 4.8. (i) $\{x$ : the order type of $x$ is regular $\} \in U$.

(ii) Let $G$ be a $\omega$-Jonsson function over $\lambda$. ( $G$ is $\omega$-Jonsson over $y$ if $G:^{\omega} y \rightarrow y$ and $G^{\prime \prime} z=y$ whenver $z \subset y$ and $|z|=|y|$.) Then we have $\left\{x: G \uparrow^{\omega} x\right.$ is $\omega$-Jonsson over $x\} \in U$.

(iii) There is an $X \in U$ so that $\operatorname{Sup} \uparrow X$ is one-to-one.

Note that normality of $U_{\alpha}$ 's is necessary in the above. Using the results proved in $\S 3$, we can show

THEOREM 4.9. For every regular $\lambda>\kappa$, there is a weakly normal minimal fine measure which does not extend $\mathrm{SCF}_{\kappa \lambda}$.

PROOF. It is clear that every normal measure is weakly normal. Hence our $U$ is weakly normal by Theorem 3.5(i). Theorem 3.3 asserts that $U$ does not extend $\mathrm{SCF}_{\kappa \lambda}$. At last $U$ is minimal by Fact 3.6, Theorem 4.1(ii), and Lemma 4.8(iii).

It is not known whether $U$ can be isomorphic to some fine measure extending $\mathrm{SCF}_{\kappa \lambda}$. We also do not know whether nonminimal weakly normal measures exist. 


\section{REFERENCES}

1. Y. Abe, Some results concerning strongly compact cardinals, J. Symbolic Logic 50 (1985), 874880.

2. A. Blass, private communication.

3. D. M. Carr, The minimal normal filter on $P_{\kappa} \lambda$, Proc. Amer. Math. Soc. 86 (1982), 316-320.

4. M. Gitik, Nonsplitting subset of $P_{\kappa}\left(\kappa^{+}\right)$, J. Symbolic Logic 50 (1985), 881-894.

5. T. J. Jech, Some combinatorial problems concerning uncountable cardinals, Ann. Math. Logic 5 (1973), 165-198.

6. J. Ketonen, Ultrafilters over measurable cardinals, Fund. Math. 77 (1973), 257-269.

7. A. Kanamori, Weakly normal filters and irregular ultrafilters, Trans. Amer. Math. Soc. 220 (1976), 393-399.

8. T. K. Menas, On strong compactness and supercompactness, Ann. Math. Logic 7 (1974), 327359.

9. _ A combinatorial property of $P_{\kappa} \lambda$, J. Symbolic Logic 41 (1976), 225-234.

10. R. M. Solovay, W. N. Reinhardt, and A. Kanamori, Strong axioms of infinity and elementary embeddings, Ann. Math. Logic 13 (1978), 73-116.

Fukushima College of Technology, taira, Kamiarakawa, Nagao 30 Iwaki, 970 JAPAN 